Research Paper

\title{
Spreading of pathological TDP-43 along corticospinal tract axons induces ALS-like phenotypes in $A \operatorname{tg} 5^{+/-}$mice
}

\author{
Rui Zhang1, Yongkang Chen2,3,8, Xinxin Wang2,3, Haiyan Tian2,3, Han Liu'2,3, Zhi Xiang2,3, Dan Qi', Jason H. \\ Huang ${ }^{4,5}$, Erxi $\mathrm{Wu}^{4,5,6,7}$, Xuebing Ding $^{2,3}{ }^{\bowtie}$, Xuejing Wang ${ }^{2,3}$ \\ 1. Department of Neurology, Xiangya Hospital, Central South University, Changsha, Hunan, 410008, China \\ 2. The First Affiliated Hospital of Zhengzhou University, Zhengzhou, Henan, 450052, China \\ 3. Institute of Parkinson and Movement Disorder, Zhengzhou University, Zhengzhou, Henan, 450052, China \\ Neuroscience Institute and Department of Neurosurgery, Baylor Scott \& White Health, Temple, Texas, 76508, USA \\ College of Medicine, Texas A\&M University, College Station, TX, 77843, USA \\ Irma Lerma Rangel College of Pharmacy, Texas A\&M University, College Station, TX, 77843, USA \\ LIVESTRONG Cancer Institutes and Department of Oncology, Dell Medical School, the University of Texas at Austin, Austin, TX, 78712, USA \\ Academy of Medical Sciences, Zhengzhou University, Zhengzhou, Henan, 450052, China \\ $\square$ Corresponding authors: erxi.wu@BSWHealth.org (Erxi Wu); fccdingxb@zzu.edu.cn (Xuebing Ding); or fccwangxj2@zzu.edu.cn (Xuejing Wang).
}

() The author(s). This is an open access article distributed under the terms of the Creative Commons Attribution License (https://creativecommons.org/licenses/by/4.0/). See http://ivyspring.com/terms for full terms and conditions.

Received: 2020.09.28; Accepted: 2020.11.23; Published: 2021.01.01

\begin{abstract}
Amyotrophic lateral sclerosis (ALS) is a progressive neurodegenerative disease, characterized by phosphorylated TDP-43 (pTDP-43)-positive inclusions in neurons and glial cells. However, the pathogenic mechanism that underlies ALS remains largely unknown. To investigate the effects of autophagy deficiency in the formation and spreading of pathological TDP-43 along corticospinal tract axons, TDP-43 preformed fibrils (PFFs) were prepared and unilaterally injected into the fifth layer of the left primary motor cortex (M1) or the left anterior horn of the seventh cervical spinal cord segment (C7) of Atg5+/- mice. After the injection of TDP-43 PFFs, the elevated levels of PTDP-43 were present in several pyramidal tract-associated regions of Atg5+/- mice. Additionally, the occurrence of spontaneous potentials detected by electromyography demonstrates evidence of lower motor neuron dysfunction in M1-TDP-43 PFFs-injected Atg5+/- mice, and prolonged central motor conduction time detected by motor evoked potentials provides evidence of upper motor neuron dysfunction in C7-TDP-43 PFFs-injected Atg5+/- mice. These results show that injection of TDP-43 PFFs into the $\mathrm{Ml}$ or $\mathrm{C7}$ of Atg5+/- mice induces the spreading of pathological TDP-43 along corticospinal tract axons in both an anterograde and retrograde manner. Importantly, TDP-43 PFFs-injected Atg5 ${ }^{+/}$mice also display ALS-like motor dysfunction. Taken together, our findings provide direct evidence that TDP-43 PFFs-injected Atg5 ${ }^{+/-}$mice exhibited ALS-like neuropathology and motor phenotypes, suggesting that autophagy deficiency promotes the formation and spreading of pathological TDP-43 in vivo.
\end{abstract}

Key words: TAR DNA-binding protein 43 (TDP-43); amyotrophic lateral sclerosis (ALS); autophagy; preformed fibrils (PFFs); Atg5 $5^{+/-}$mice

\section{Introduction}

Amyotrophic lateral sclerosis (ALS) is a progressive neurodegenerative disease [1-4], characterized by phosphorylated TDP-43 (pTDP-43)-positive inclusions in neurons and glial cells [5-7]. However, the pathogenic mechanism that underlies ALS remains unclear [8]. Postmortem histological studies have identified stereotypical spreading patterns of pTDP-43, and these findings suggest that pTDP-43-positive inclusions may spread along the axons in central nervous system $[9,10]$. In addition, a few in vitro studies suggest that pathological TDP-43 can be transmitted from cell to cell along axon in a self-templating manner [11-16]. Furukawa et al. reported that sarkosyl-insoluble TDP-43 fibrils were able to evoke TDP-43 aggregation in HEK293T cells, and this seeding reaction could reproduce ubiquitinated TDP-43 aggregates in cells [12]. Furthermore, Nonaka et al. found that sarkosyl-insoluble TDP-43 extracted from ALS or frontotemporal lobar degeneration (FTLD) brains 
induces TDP-43 aggregation in SH-SY5Y cells, and these cells subsequently formed ubiquitinated, phosphorylated, insoluble cytoplasmic TDP-43 inclusions in a self-templating manner, thus demonstrated seeded aggregation of TDP-43 [13]. In addition, TDP-43 oligomers may spread intercellularly across axon terminals of primary cortical mouse neurons [14]. In vivo, by using a zebrafish model, Svahn and his colleagues first demonstrated the nucleo-cytoplasmic transport of TDP-43 [17]. Until recently, Porta et al. showed that injection of pathological TDP-43 derived from FTLD-TDP brains could lead to the formation and trans-neuronal spreading of TDP-43 pathology in both CamKIIa-hTDP43 ${ }_{\text {NLSm }}$ mice and non-transgenic mice [18]. We speculate that the spreading of pathological TDP-43 via corticospinal tract may be one of the underlying mechanisms of ALS.

In our present study, TDP-43 preformed fibrils (PFFs) were prepared and unilaterally injected into the fifth layer of the left primary motor cortex (M1) or the left anterior horn of the seventh cervical spinal cord segment (C7) of Atg5 $5^{+/}$mice. After the injection of TDP-43 PFFs, the elevated levels of pTDP-43 were present in several pyramidal tract-associated regions of Atg5 $5^{+/}$mice. In addition, the occurrence of spontaneous potentials detected by electromyography (EMG) demonstrates evidence of lower motor neuron (LMN) dysfunction in M1-TDP-43 PFFs-injected Atg5 $5^{+/-}$mice, and prolonged central motor conduction time (CMCT) detected by motor evoked potentials (MEPs) suggests upper motor neuron (UMN) lesion in C7-TDP-43 PFFs-injected Atg5 $5^{+/-}$mice. Moreover, behavioral analysis indicates that both M1- and C7-TDP-43 PFFs-injected Atg5 ${ }^{+/}$- mice developed an ALS-like syndrome in a time-dependent manner.

\section{Materials and methods}

\section{Animals}

Eight-week-old male B6: 129-Atg5<tm1Nmz> (RBRC02231) (Atg5+/-) and C57BL/6 mice weighing 18-20 g were used. Animals were kept under standard laboratory conditions with free access to standard laboratory food and water $\left(21^{\circ} \mathrm{C}, 12 \mathrm{~h} / 12 \mathrm{~h}\right.$ light-dark cycle). All experiments were performed in accordance with the Guide for the Care and Use of Laboratory Animals. The protocols were approved by the Institutional Ethics Committees of the Zhengzhou University.

\section{TDP-43 PFFs preparation and Electron Microscopy Imaging}

TDP-43 PFFs were prepared as previously reported [11, 12]. Monomeric TDP-43 (Proteintech,
Wuhan, China) was resuspended in sterile water at concentration of $0.5 \mathrm{mg} / \mathrm{ml}$. To obtain TDP-43 PFFs, the samples were incubated at $37^{\circ} \mathrm{C}$ for 2 hours with agitation at $600 \mathrm{rpm}$ in an Eppendorf Thermomixer. For electron microscopy imaging, a $5 \mu \mathrm{l}$ sample was absorbed onto carbon/formvar-coated 150 mesh copper grids (Yasheng Electronics Technology Co, Ltd., Zhengzhou, China) for 30 seconds and then stained with $5 \mu \mathrm{l}$ of $2 \%$ uranyl acetate for 30 seconds. After staining, the grids were rinsed briefly in distilled water. The excess water was removed with filter paper, and the grids were air-dried prior to analysis by transmission electron microscopy (JEOL USA, Inc., Peabody, MA, USA).

\section{Stereotaxic surgery on mice}

One hundred and twenty Atg5 $5^{+/}$mice were divided into 4 groups equally: M1-TDP-43 PFFs injection group, M1-PBS injection group, C7-TDP-43 PFFs injection group, and C7-PBS injection group. The mice were deeply anesthetized using isoflurane during the whole surgical procedure and immobilized in a stereotaxic frame (David Kopf Instruments, Tujunga, CA, USA). Unilateral injections were made into the fifth layer of the left M1 or left anterior horn of C7 at the following coordinates: M1: anteroposterior, $0.7 \mathrm{~mm}$; mediolateral, $1.5 \mathrm{~mm}$; dorsoventral, $1.4 \mathrm{~mm}$; and C7: mediolateral, $0.8 \mathrm{~mm}$; dorsoventral, $1.2 \mathrm{~mm}$. Each injection site received $5 \mu \mathrm{l}$ of TDP- 43 PFFs or PBS at a rate of $0.2 \mu \mathrm{l} /$ minute using a Hamilton Syringe (Hamilton, NV, USA).

\section{Survival analysis}

Date and cause of death were recorded for each mouse. For ethical reasons, animals were closely monitored and sacrificed as moribund prior to actual death using criteria for severe illness. To determine the duration of survival reliably and humanely, the moribund state, defined as the inability of mice to right themselves 30 seconds after being placed on their side, was used. The moribund mice were scored as "dead" and were euthanized.

\section{Immunohistochemistry}

After euthanasia and perfusion, the brain and spinal cord from perfused animals were fixed in 30\% sucrose solution containing $4 \%$ paraformaldehyde. Tissues were embedded in paraffin blocks, cut into 4 $\mu \mathrm{m}$ using a Rotary Microtome (Leica RM2235, Leica, Nussloch, Germany), and mounted on glass slides. Sections of spinal cord and brain tissues were then deparaffinized and rehydrated in a graded ethanol series. Immunohistochemistry was performed as previously described [19]. Briefly, tissues were incubated in 3\% hydrogen peroxide solution and then subjected to antigen retrieval at $100{ }^{\circ} \mathrm{C}$ for 10 minutes 
in citrate buffer. Tissues were blocked in normal goat serum for 20 minutes at room temperature and then incubated with anti-pTDP-43 (phosphorylated at Ser409/Ser410) antibody (mouse, Millipore, 1:800) at 4 ${ }^{\circ} \mathrm{C}$ for 24 hours. Then, the labeling was detected using a Streptavidin-Peroxidase kit (Bioss, China) and visualized with diaminobenzidine. Images were captured using an Olympus IX51 microscope mounted with a DP71 Olympus digital camera.

\section{Preparation of mouse tissue lysates and immunoblotting}

Frozen spinal cord or brain samples were thawed on ice and sonicated in $5 \times \mathrm{v} / \mathrm{w}$ RIPA buffer (50 mM Tris, $150 \mathrm{mM} \mathrm{NaCl}, 1 \%$ NP-40, $5 \mathrm{mM}$ EDTA, $0.5 \%$ sodium deoxycholate, and $0.1 \%$ SDS, $\mathrm{pH} 8.0$ ) containing protease inhibitor cocktails (Thermo Fisher Scientific). Samples were centrifuged for 30 minutes at $4{ }^{\circ} \mathrm{C}, 100,000 \mathrm{~g}$. The supernatant was taken as RIPA-soluble fractions. The pellet was sonicated with $5 \times \mathrm{v} / \mathrm{w}$ RIPA buffer as above. Then, the pellet was sonicated in $2 \times \mathrm{v} / \mathrm{w}$ urea buffer $(7 \mathrm{M}$ urea, $2 \mathrm{M}$ thiourea, $4 \%$ CHAPS, and $30 \mathrm{mM}$ Tris, $\mathrm{pH} 8.5$ ) and centrifuged for 30 minutes at $22{ }^{\circ} \mathrm{C}, 100,000 \mathrm{~g}$. This supernatant was collected as the RIPA-insoluble/urea-soluble fractions. Samples were analyzed by $10 \%$ SDS-PAGE with polyvinylidene fluoride (PVDF) membranes. Following transfer to the PVDF membranes, the immunoblot was sequentially probed with antibodies that recognize pTDP-43 (mouse, Millipore, 1:800) or LC3A/B (rabbit, Cell Signaling Technology, 1:1000). The densities of the proteins were normalized to GAPDH.

\section{Needle EMGs}

Mice were anesthetized with isoflurane and placed in the prone position on a thermostatically controlled warming plate to maintain body temperature at $37{ }^{\circ} \mathrm{C}$. A standard EMG apparatus (MEB-2306C, Nihon Kohden Corporation, Tokyo, Japan) was applied. To determine UMN and LMN deficits, we collected motor unit action potentials (MUAPs) and spontaneous activities including fibrillation potentials, positive sharp waves and fasciculation potentials. A sweep speed of $10 \mathrm{~ms} / \mathrm{div}$, a gain of $100 \mathrm{uv} /$ div and a band pass filter with low and high cut-off frequency of 20 and $10,000 \mathrm{~Hz}$ were performed. The mice were electrically grounded through a disposable needle electrode implanted subcutaneously at the abdominal wall. Next, we used concentric needles $(25.0 \mathrm{~mm} \times 0.3 \mathrm{~mm}$, Technomed Europe, Beek, Netherlands) to detect the following muscles: bilateral biceps brachii, bilateral T10 paraspinal muscles, bilateral tibialis anterior, and bilateral gastrocnemius. Spontaneous activities were recorded for 120 seconds and MUAPs were recorded at different sites of each muscle until 20 MUAPs were obtained.

\section{Electrical stimulation and recordings of MEPs}

Cortical MEP (cMEP) and spinal MEP (sMEP) were obtained using two monopolar needle electrodes. For cMEP, the cathode was positioned at the midline of the interaural line through the scalp; the anode was positioned $4-5 \mathrm{~mm}$ lateral and anterior to the cathode. For sMEP, two monopolar needle electrodes were inserted into C7, close to the emergence of the spinal nerve root. cMEP and sMEP were recorded with the active needle electrode inserted into the muscle of the forelimb footpad and reference needle electrode inserted under the skin of the second digit. The onset of the first, generally negative, deflection was taken as the MEP latency. The MEP with the shortest latency was considered as the final evaluation criteria of MEP latency, the peak-to-peak amplitude of the motor response was used for the mean MEP amplitude. The CMCT is the propagation time between motor cortex and spinal cord, which is the difference between the cMEP latency and the sMEP latency.

\section{H\&E staining}

Biopsied biceps brachii were dissected out from the forelimb of an anesthetized animal and immersed immediately in isopentane cooled in liquid nitrogen. Serial frozen sections were cut at $10 \mu \mathrm{m}$ thickness and stained by hematoxylin and eosin (H\&E). All steps were performed according to the standard procedures [20].

\section{Behavioral tests}

To evaluate behavioral performance, 15 mice from each group were weighed weekly and underwent a battery of behavioral tests every two weeks starting from the second month post-surgery. Baseline performance was measured before surgery. The experimenter was blinded to the treatment group for all behavioral tests.

\section{Rotarod test}

Rotarod test was performed using the rotarod apparatus (Rotarod YLS-4C; YiYan Science and Technology Development Co., Ltd. Shandong, China). The test began by placing the mice on the rod rotating at $30 \mathrm{rpm}$. The latency to fall off the rotarod within the maximum time (180 seconds) was recorded as a measurement of the competence of their motor function. Three trials were performed per day with a 15-minute inter-trial interval. The mean latency to fall off the rotarod was calculated for the data analysis. 

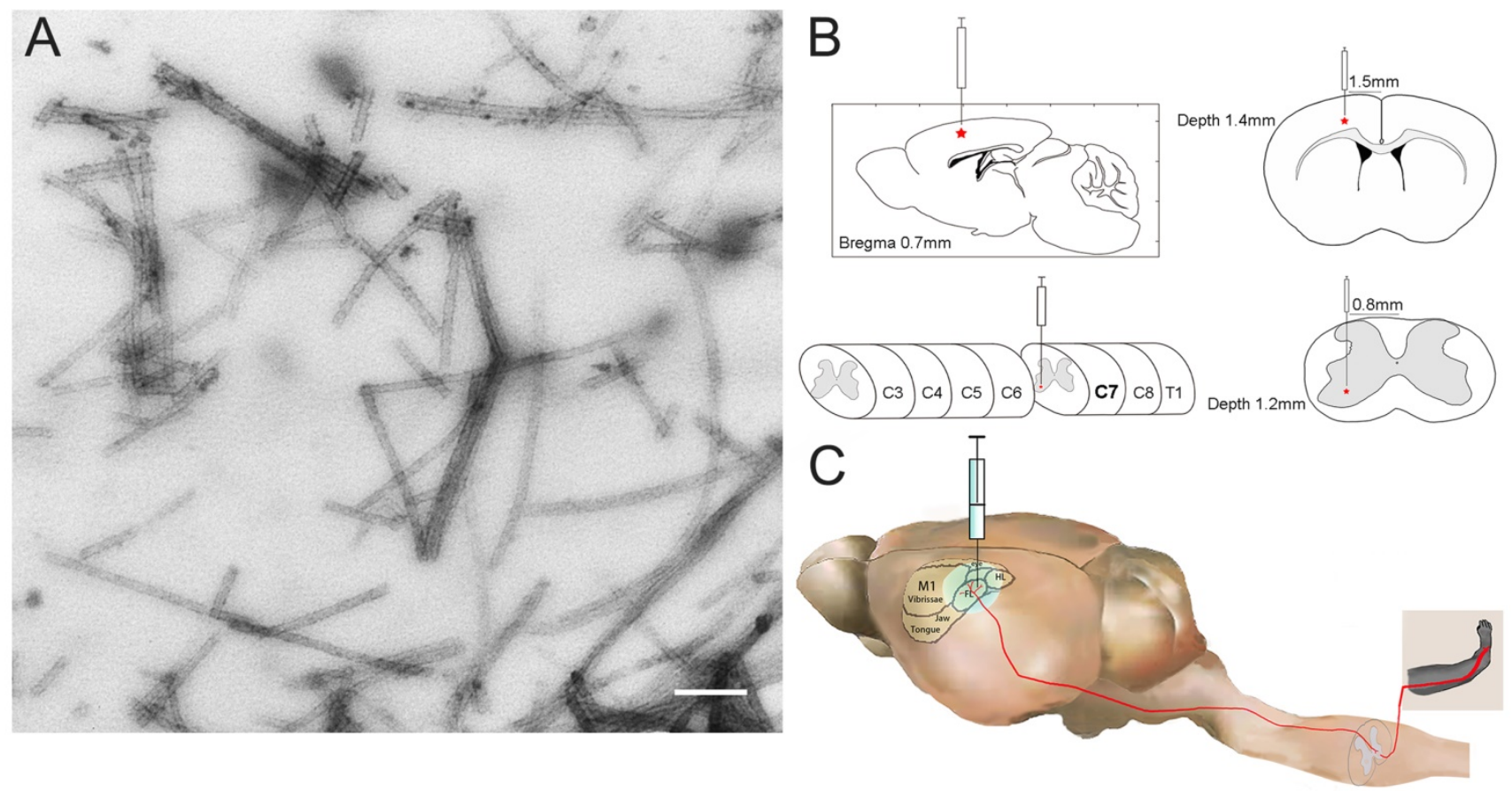

Figure 1. The construction of MI- and C7-TDP-43 PFFs-injected Atg5+/- mouse model. A. Negatively stained transmission electron micrograph of TDP-43 PFFs. Scale bar = 200 $\mathrm{nm}$. B. The schematic diagram of MI- and C7-TDP-43 PFFs/PBS-injected Atg5+/- mouse model in the coronal and axial position. C. The nerve conduction pathway from the forelimb region of $\mathrm{MI}$ to the skeletal muscles of the forelimb.

\section{Hanging wire test}

The hanging wire test was performed to evaluate general muscle strength. Each mouse was placed on a horizontally positioned screen with many $1 \mathrm{~cm} \times 1 \mathrm{~cm}$ grids. The period until the mouse left the screen was recorded, with a maximum time of 180 seconds. Three trials were performed per day, and the mean latency was recorded for the data analysis.

\section{Footprint test}

To obtain footprints, paws of the mice were painted with two different non-toxic paints (fore-paws in red and hind-paws in green). The mice were then allowed to walk along a restricted cardboard tunnel ( $50 \mathrm{~cm}$ long, $5 \mathrm{~cm}$ wide, $10 \mathrm{~cm}$ high) into an enclosed box and a sheet of white paper was placed on the floor of the tunnel, and one set of footprints were collected for each mouse. Three steps from the middle portion of each run were measured for the following parameters $(\mathrm{cm})$ : (1) stride length (front and hind legs). (2) The front- and hind-base width. The mean value of each set of values was used for the data analysis.

\section{Statistical analysis}

The statistical analyses were performed using SPSS 24.0 (IBM, Armonk, New York, USA). Behavioral and electrophysiological data of mice were presented as mean \pm standard deviation (SD), and two-way ANOVA was employed for comparison of multiple groups. Disease onset and survival statistics were performed by Kaplan-Meier survival curves and the data were analyzed using the log-rank test, generating a $X^{2}$ value to test for significance. For all statistical tests, significance was taken as ${ }^{*} p<0.05$, ${ }^{* *} p$ $<0.01,{ }^{* * *} p<0.001$.

\section{Results}

\section{TDP-43 PFFs induce PTDP-43-immunoreactive deposits in situ}

To investigate whether pathological TDP-43 could spread along corticospinal tract axons, we injected TDP-43 PFFs (Figure 1A) into the fifth layer of the left M1 or the left anterior horn of C7 of Atg5 $5^{+-}$ mice (Figure 1B). Diagrams of estimated conduction pathway for pathological TDP-43 spreading were shown in Figure 1C. Firstly, pTDP-43 immunoactivity was detected at the injection sites using an anti-pTDP-43 (phosphorylated at Ser409/Ser410) antibody. As early as 2 months post-injection (mpi), pTDP-43-immunoreactive (pTDP-43-ir) pathology was detected in the neurons and glial cells in the second, third and fifth layers of the M1 around the injection region of M1-TDP-43 PFFs-injected Atg5 $5^{+/}$ mice (Figure 2Aa, 2Ac, and 2Ae); however, the staining was not observed on the contralateral side (Figure 2Ab, 2Ad, and 2Af). In the cervical enlargement of C7-TDP-43 PFFs-injected Atg5+/- mice, pTDP-43-ir pathology was found in the neurons of the left spinal cord anterior horn adjacent to the injection 
sites at 2 mpi (Figure 2Ea and 2Eb). No pTDP-43-ir pathology was detected in the age-matched PBS-injected Atg5 $5^{+/-}$mice (Figure 3A and 3E). Thus, these data indicated that TDP-43 PFFs could induce the formation of pTDP-43 pathology in vivo. These data supported that the injection of TDP-43 PFFs into the brain or spinal cord induce pTDP-43-ir pathology deposits in situ.

\section{Pathological TDP-43 spreads along corticospinal tract axons in both anterograde and retrograde manner}

To demonstrate whether spreading of pathological TDP-43 via corticospinal tract induces ALS-like neuropathology, we next evaluated the pTDP-43 pathology in different sections along the corticospinal tract at different time points in Atg5 $5^{+/-}$ mice.

At about $6 \mathrm{mpi}$, pTDP-43-ir staining was detected in the hippocampus (Figure 2B), medulla oblongata (Figure 2C), and cervical enlargement (Figure 2D) of M1-TDP-43 PFFs-injected Atg $5^{+/-}$mice. In the cortex, neuronal and glial cytoplasmic pTDP-43-ir pathology mainly deposited in the fifth layer of the left M1 (Figure 2Ba and 2Be), while no pTDP-43 pathology was observed on the contralateral side (Figure $\mathbf{2} \mathbf{B b}$ and $\mathbf{2} \mathbf{B f}$ ). In the CA1 region of the hippocampus, the positive neuronal and glial cytoplasmic pTDP-43 staining was more intense on the left side (Figure $\mathbf{2 B C}$ and $\mathbf{2 B g}$ ) than the contralateral side (Figure 2Bd and 2Bh). In medulla oblongata, the positive neuronal and glial cytoplasmic pTDP-43 pathology was detected in both sides of hypoglossal nucleus (Figure $2 \mathrm{Ca}, 2 \mathrm{Cb}, 2 \mathrm{Ce}$, and $2 \mathrm{Cf}$ ) and the pTDP-43-ir nerve fibers in the left corticospinal tract (Figure $2 \mathrm{Cc}$ and $\mathbf{2} \mathrm{Cg}$ ), and the pTDP-43 staining was stronger in the left side of the hypoglossal nucleus (Figure $2 \mathrm{Ca}$ and $2 \mathrm{Ce}$ ), however, the pTDP-43-ir staining was not detected in the contralateral side of the corticospinal tract (Figure 2Cd and 2Ch). Furthermore, neuronal and glial cytoplasmic pTDP-43 was deposited in the right side of anterior horn of the cervical enlargement (Figure 2Da and 2Db). However, no pTDP-43 pathology was detected in both sides of M1-PBS-injected Atg $5^{+/-}$mice (Figure 3B-D).

In C7-TDP-43 PFFs-injected Atg5 ${ }^{+/}$mice, neuronal and glial cytoplasmic pTDP-43 staining was more frequently observed in the right side of the hypoglossal nucleus (Figure $\mathbf{2} \mathbf{F b}$ and $\mathbf{2 F f}$ ), CA1 region of hippocampus (Figure $2 \mathrm{Gd}$ and $2 \mathrm{Gh}$ ), and the fifth layer of the M1 (Figure 2Gb, 2Gf, $2 \mathbf{H b}, 2 \mathbf{H d}$, and $\mathbf{2 H f}$ ) compared to the left side (Figure 2Fa, 2Fc, 2Fe, 2Fg, $2 \mathrm{Ga}, 2 \mathrm{Gc}, 2 \mathrm{Ge}, 2 \mathrm{Gg}, 2 \mathrm{Ha}, 2 \mathrm{Hc}$, and $2 \mathrm{He}$ ) at about 5 mpi. The pTDP-43-ir nerve fibers were detected in the left corticospinal tract at about 5 mpi (Figure 2 Fd and 2Fh). As with M1-PBS-injected Atg5 ${ }^{+/-}$mice, no pTDP-43 pathology was detected in both sides of C7-PBS-injected Atg5 $5^{+/-}$mice (Figure 3F-H). In addition, we also injected TDP-43 PFFs into the C7 of C57BL/ 6 mice. However, the formation of TDP-43-ir neuronal and glial cytoplasmic pathology in the fifth layer of the M1 was not detected until 18 months (Figure 4). Taken together, these data suggest that the injection of TDP-43 PFFs into motor cortex or ventricornu of $\operatorname{Atg} 5^{+/-}$mice induces the spreading of TDP-43 pathology along corticospinal tract axons anterogradely and retrogradely in vivo.

To evaluate whether autophagy deficiency can affect the aggregation of pathological TDP-43, we first detected LC3 expression levels in M1, hippocampus, medulla oblongata, and cervical enlargement of C57BL/ 6 and Atg5 $5^{+/-}$mice, the results showed that LC3A/B-II/LC3A/B-I levels were significantly decreased in these regions of adult $\mathrm{Atg} 5^{+/-}$mice compared with age-matched C57BL/ 6 mice (Figure 5A-D). To further demonstrate whether there was an increase of pTDP-43 pathology in TDP-43 PFFs-injected Atg5 $5^{+/}$mice, immunoblotting analysis was also performed in the abovementioned regions of M1-TDP-43 PFFs- and M1-PBS-injected Atg5 ${ }^{+/-}$mice at $6 \mathrm{mpi}$. In the soluble fractions, no significant difference of pTDP-43 levels was found between M1-TDP-43 PFFs- and M1-PBS-injected Atg5 ${ }^{+/-}$mice (Figure 5E-H). Nevertheless, in the insoluble fractions, pTDP-43 levels were increased in all examined M1-TDP-43 PFFs-injected Atg5 $5^{+/-}$mice, while it was faintly detected in age-matched PBS-injected Atg5 $5^{+/-}$mice (Figure 5E-H). The same results were also found in C7-TDP-43 PFFs-injected Atg5 $5^{+/-}$mice at $5 \mathrm{mpi}$ (data not shown). These results support that the injection of TDP-43 PFFs into M1 or C7 of $\mathrm{Atg} 5^{+/-}$mice could induce the spreading of pathological TDP-43 along corticospinal tract axons anterogradely and retrogradely.

\section{M1- and C7-TDP-43 PFFs-injected Atg5 ${ }^{+/-}$mice exhibit ALS-like neurophysiological phenotypes}

Besides the morphological evidence of TDP- 43 PFFs-induced pathology, we further investigated whether the M1- and C7-TDP-43 PFFs-injected Atg5 ${ }^{+/-}$ mice exhibited any neurophysiological abnormality. Spontaneous activity, motor unit potential recruitment, and MUAPs were used to quantify LMN dysfunction. MEPs were used to evaluate potential impairment of the corticospinal tract. Firstly, needle EMGs were recorded from the biceps brachialis, T10 paraspinals, tibialis anterior, and gastrocnemius muscles. In M1-TDP-43 PFFs-injected mice, abnormal 
spontaneous activity including fibrillation potentials (Figure 6A), fasciculation potentials (Figure 6B), and positive sharp waves (Figure 6C) were detected on the right side of biceps brachialis, T10 paraspinals, tibialis anterior, and gastrocnemius muscles as early as $2 \mathrm{mpi}$. At $5 \mathrm{mpi}$, abnormal spontaneous activities were found in both side of biceps brachialis, T10 paraspinals, tibialis anterior, and gastrocnemius muscles, and there was no significantly difference between the two sides (Figure 6D). Significantly, the abnormal spontaneous activities were detected in the right biceps brachialis of all the M1-TDP-43 PFFs-injected mice, however, the M1-PBS-injected Atg $5^{+/}$mice only showed mild abnormal spontaneous activity in right biceps brachialis muscles at $7 \mathrm{mpi}$ (Figure 6D). The frequency of abnormal spontaneous activity was developed in a time-dependent manner as shown in Figure 6D. Secondly, we collected 20 MUAPs in each mouse for the motor unit measurement. The findings showed a significantly increase in the mean amplitude and duration of MUAPs at 2, 5, and 7 mpi in M1-TDP-43 PFFs-injected Atg5 $5^{+/-}$mice, compared to age-matched M1-PBS-injected Atg5 $5^{+/-}$mice (Figure 6E and 6F). Furthermore, we performed a biopsy in both sides of the biceps brachialis in M1-TDP-43 PFFs-injected Atg $5^{+/-}$mice at $7 \mathrm{mpi}$. H\&E staining showed muscle atrophy, round muscle fibers, and muscle fibers with central nuclei in the right biceps brachialis (Figure 6K), while no morphological changes were found in the left biceps brachialis (Figure 6L). Taken together, these findings suggest LMN dysfunction in M1-TDP-43 PFFs-injected Atg5 $5^{+/-}$mice.
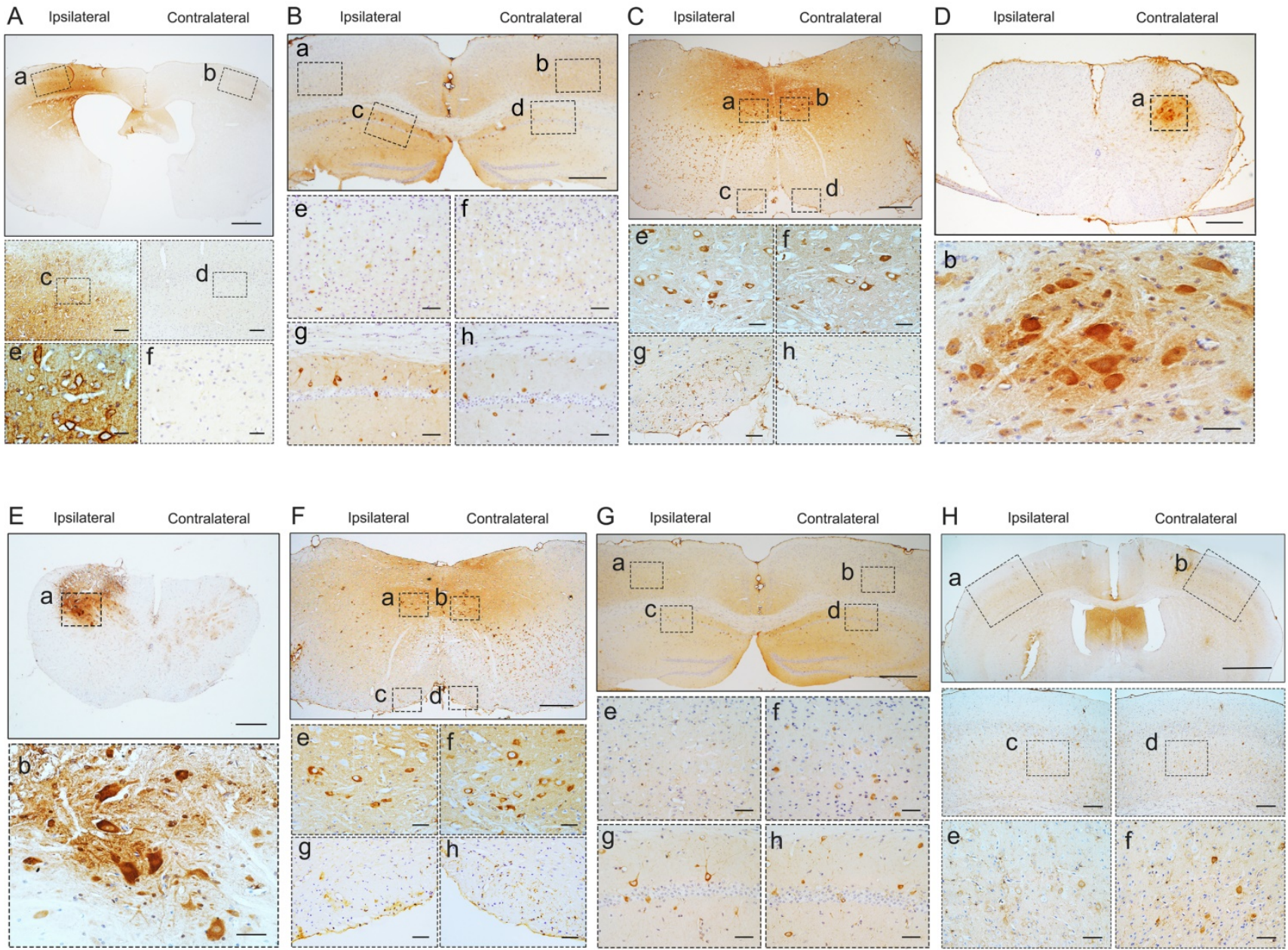

Figure 2. Pathological TDP-43 spread along corticospinal tract axons anterogradely and retrogradely. PTDP-43 staining was performed in MI (A) at 2 mpi, hippocampus (B), medulla oblongata (C), and cervical enlargement (D) at 6 mpi of Ml-TDP-43 PFFs-injected Atg5+/- mice. A. In the second, third and fifth layer of the MI, pTDP-43-ir in neurons and glial cells was more abundant in the left side (a, c, and e) than the contralateral side (b, d, and f). Scale bars: a-b, $1000 \mu \mathrm{m} ; \mathrm{c}-\mathrm{d}, 100 \mu \mathrm{m}$; e-f, $25 \mu \mathrm{m}$. B. In the fifth layer of Ml and CAl region of hippocampus, neuronal and glial cytoplasmic pTDP-43-ir was also more severe in the left side (a, c, e, and g) than contralateral side (b, d, f, and h). Scale bars: a-d, $1000 \mu \mathrm{m}$; e-h, $100 \mu \mathrm{m}$. C. In medulla oblongata, neuronal and glial cytoplasmic pTDP-43-ir was stronger in the left side of the hypoglossal nucleus (a and e) and pyramidal tract (c and g) than the contralateral side (b, $\mathrm{f}, \mathrm{d}$ and $\mathrm{h}$ ). Scale bars: a-d, $500 \mu \mathrm{m} ; \mathrm{e}-\mathrm{h}, 50 \mu \mathrm{m}$. D. In cervical enlargement, neuronal and glial cytoplasmic pTDP-43-ir was mainly deposited in the right side of the anterior horn (a and b). Scale bars: a, $500 \mu \mathrm{m}$; b, $50 \mu \mathrm{m}$. PTDP-43 staining was performed in cervical enlargement (E) at 2 mpi, medulla oblongata (F), hippocampus (G), and Ml (H) at 5 mpi of C7-TDP-43 PFFs-injected Atg $5^{+/-}$mice. E. In cervical enlargement, pTDP-43-ir was predominantly deposited in the neurons of the left anterior horn (a and b). Scale bars: a, $500 \mu \mathrm{m}$; b, $50 \mu \mathrm{m}$. F. In medulla oblongata, neuronal and glial cytoplasmic pTDP-43-ir was stronger in the right side of hypoglossal nucleus (b and f) and py (d and h) than left side (a, c, e and g). Scale bars: a-d, $500 \mu \mathrm{m} ; \mathrm{e}-\mathrm{h}, 50 \mu \mathrm{m}$. G. In the fifth layer of the MI and CAl region of the hippocampus, neuronal and glial cytoplasmic PTDP-43-ir was also more severe in the right side (b, d, $f$, and $h$ ) than left side (a, $c$, e, and g). Scale bars: a-d, $1000 \mu \mathrm{m}$; e-h, $100 \mu \mathrm{m}$. H. In the fifth layer of MI, neuronal and glial cytoplasmic pTDP-43-ir was more abundant in the right side (b, d, and f) than left side (a, c, and e). Scale bars: a-b, $1000 \mu \mathrm{m} ; \mathrm{c}-\mathrm{d}, 100 \mu \mathrm{m}$; e-f, $25 \mu \mathrm{m}$. 

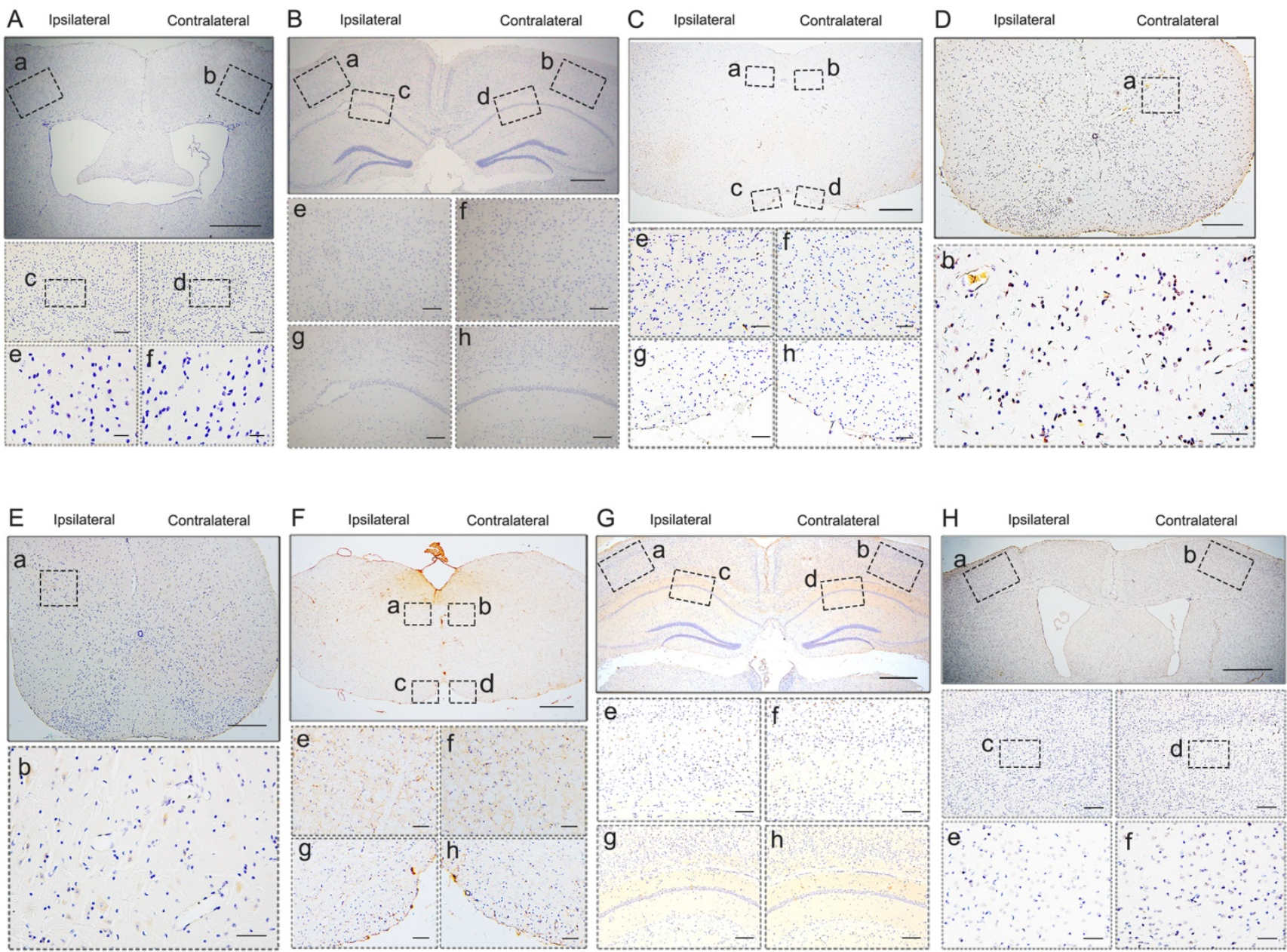

Figure 3. pTDP-43 staining in MI- and C7-PBS-injected Atg5 ${ }^{+/}$mice. A-H. No pTDP-43 pathology was found in both sides of MI- and C7-PBS-injected Atg5 ${ }^{+/-}$mice (compared with Figure 2).
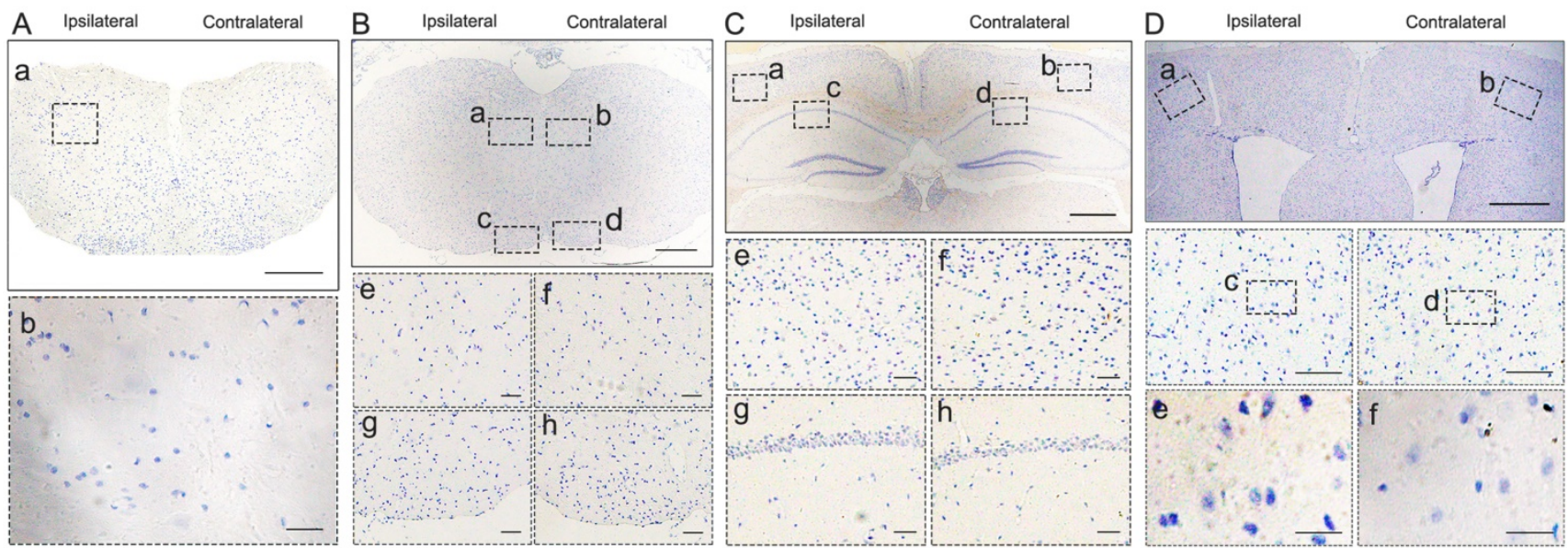

Figure 4. pTDP-43 staining in C7-TDP-43 PFFs-injected C57BL/6 mice. A-D. No pTDP-43 pathology was found in both sides of C7-TDP-43 PFFs-injected Atg5+/- at 18 mpi (compared with Figure 2E-H).

Furthermore, we performed cMEP and sMEP in C7-TDP-43 PFFs-injected Atg5 $5^{+/-}$mice to quantify $\mathrm{UMN}$ impairment (Figure 6G1, 6G2, 6H1 and 6H2). At about $2 \mathrm{mpi}$, CMCT was significantly prolonged in the injection side of C7-TDP-43 PFFs-injected Atg5+/mice compared to age-matched C7-PBS-injected
Atg5 $5^{+/-}$mice $(3.1 \pm 0.35 \mathrm{~ms}$ versus $2.44 \pm 0.36 \mathrm{~ms}, p<$ $0.05)$, and the CMCT increased in a time-dependent manner (Figure 6I) in C7-TDP-43 PFFs-injected Atg $5^{+/}$- mice. Moreover, cMEP amplitude decreased in C7-TDP-43 PFFs-injected Atg5 $/ /-$ mice compared to age-matched C7-PBS-injected Atg5 $5^{+/-}$mice (4.57 \pm 1.14 
$\mathrm{mV}$ versus $8.23 \pm 2.10 \mathrm{mV}, p<0.001$ ) at about $2 \mathrm{mpi}$, and this decline became more significant over time (Figure 6J). At the end stage, responses to cortical stimulation were absent in C7-TDP-43 PFFs-injected Atg $5^{+/-}$mice, indicating devastating damage of the corticospinal tract. Taken together, the quantitative analyses of CMCT and cMEP amplitude indicated UMN dysfunction in C7-TDP-43 PFFs-injected Atg5+/mice.
A

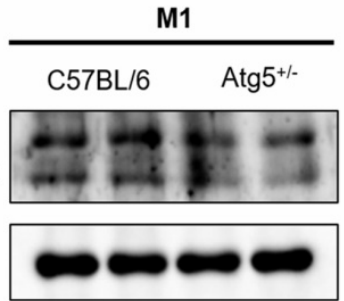

C

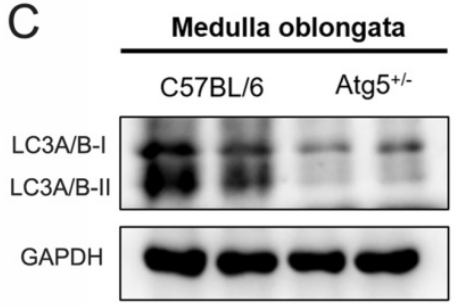

E

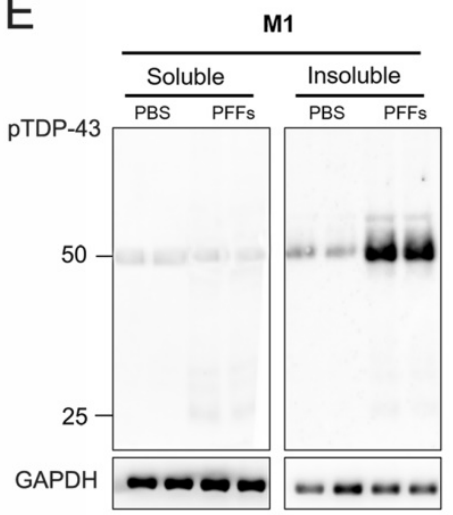

G

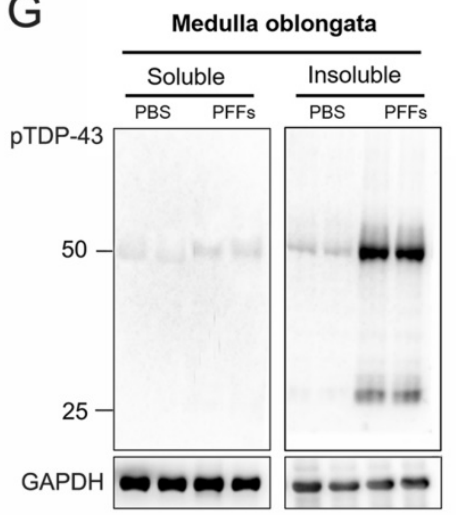

- C57BL/6

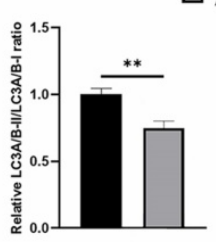

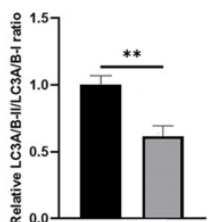
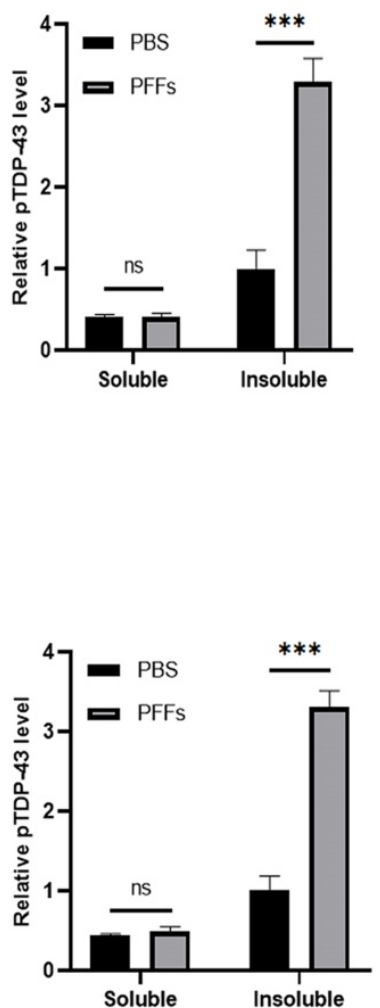

B
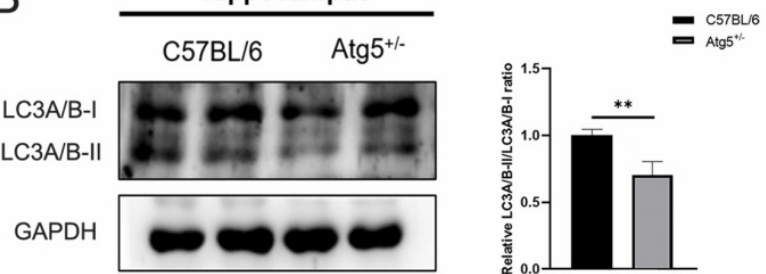

$\mathrm{D}$

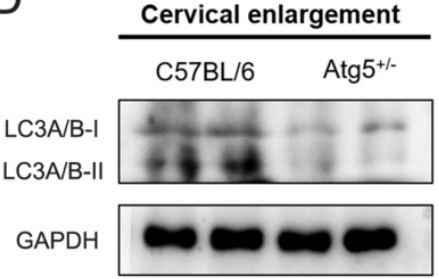
= ${ }_{\text {Atg } 5 \%}$

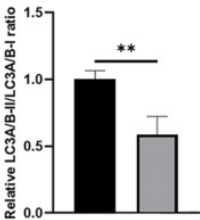

$\mathrm{F}$
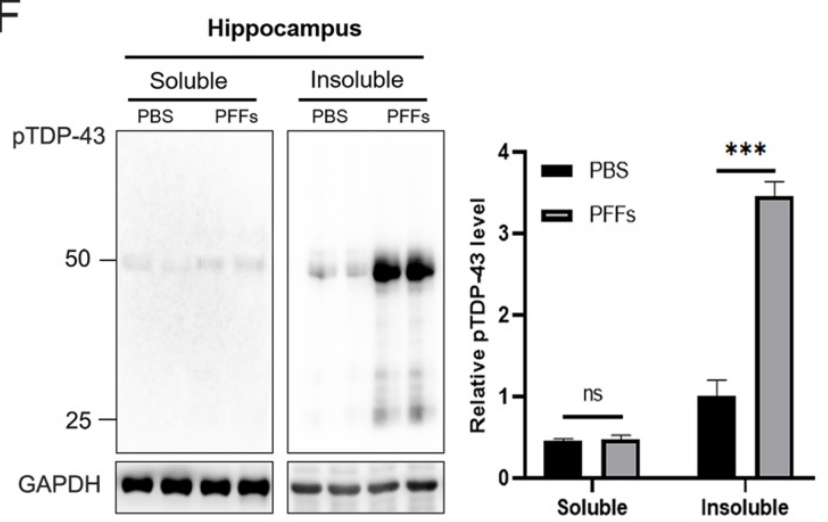

$\mathrm{H}$
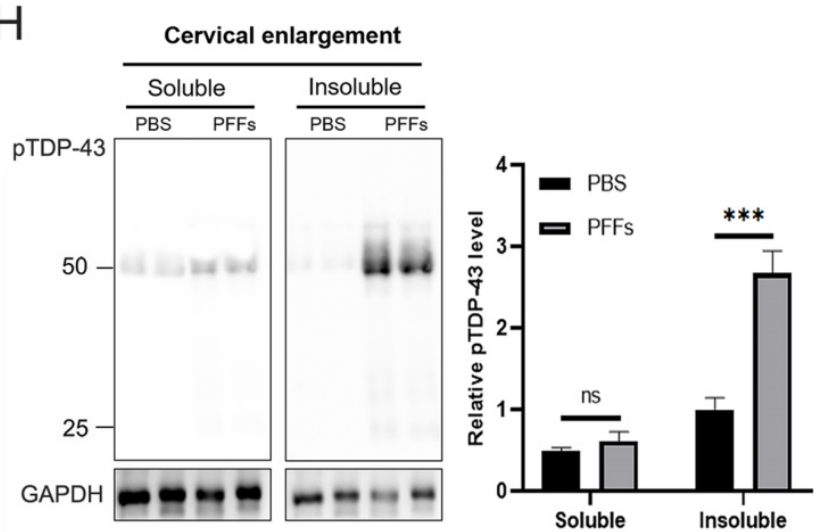

Figure 5. Immunoblotting analysis of LC3A/B and pTDP-43. A-D. Representative immunoblotting of LC $3 A / B$ in $M 1$ ( $A$ ), hippocampus (B), medulla oblongata (C), and cervical enlargement (D) of adult Atg5+/- mice and age-matched C57BL/6 mice. E-H. Representative immunoblotting of pTDP-43 in the RIPA-soluble and insoluble fractions of MI (E), hippocampus $(\mathrm{F})$, medulla oblongata $(\mathrm{G})$, and cervical enlargement $(\mathrm{H})$ of M1-TDP-43 PFFs-injected Atg5 $5^{+/-}$mice (at 6 mpi) and age-matched M1-PBS- injected Atg5 ${ }^{+/-}$mice. Blots were probed for GAPDH as loading control (bottom). 

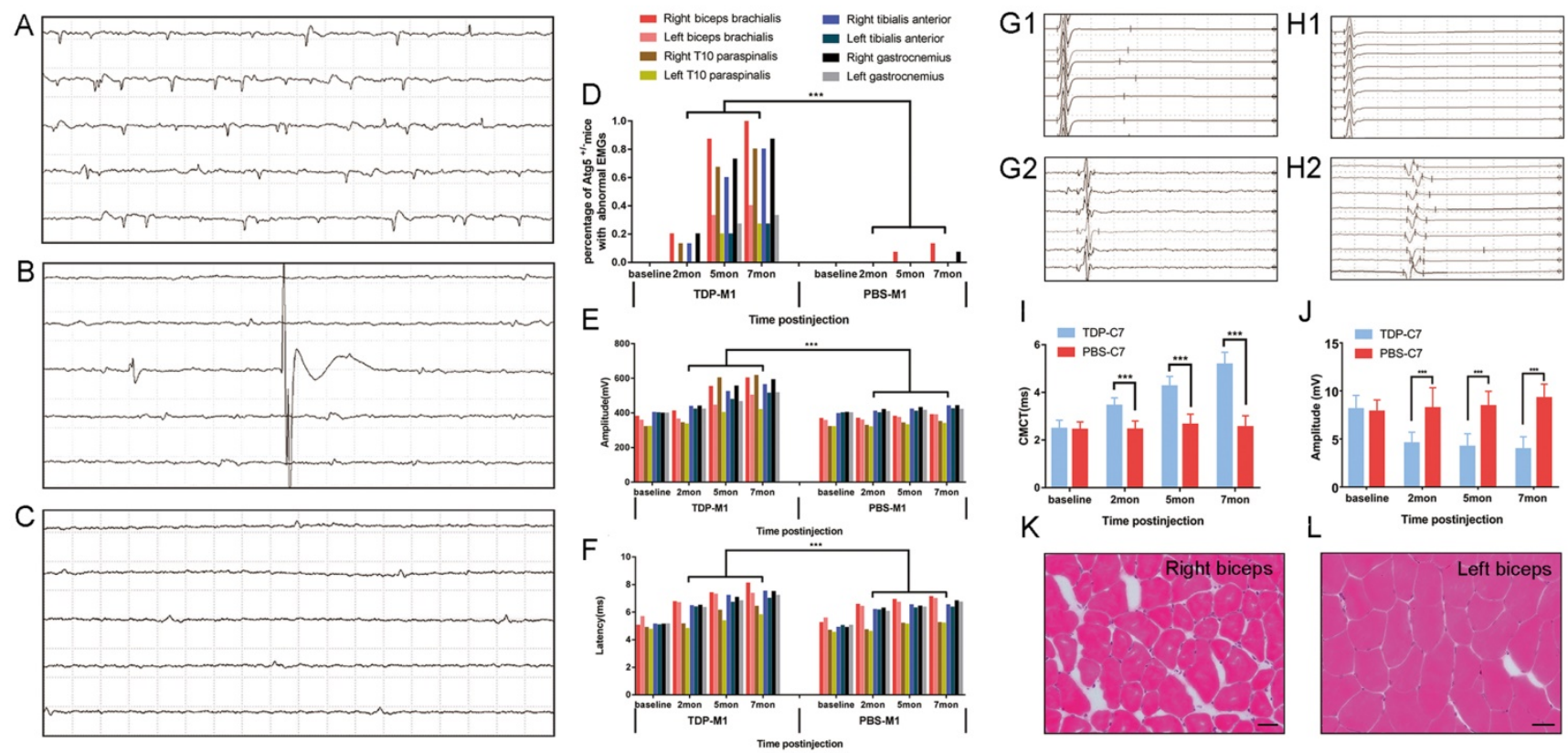

Figure 6. TDP-43 PFFs-injected Atg5+/- mice developed ALS-like changes in neurophysiology and skeletal muscle morphology. A-C. At 2 mpi, spontaneous potentials including fibrillation potentials (A), fasciculation potentials (B), and positive sharp waves (C) were detected in M1-TDP-43 PFFs-injected Atg5+/- mice. D. The frequencies of spontaneous potentials were much higher in MI-TDP-43 PFFs-injected mice compared to Ml-PBS-injected Atg5+-mice. E-F. The amplitude (E) and latency of MUAPs (F) were increased in MI-TDP-43 PFFs-injected mice compared to M1-PBS-injected Atg5+/-mice, $p<0.05$. G-H. Left-sMEP (L-sMEP) (G1 and H1) and Right-cMEP (R-cMEP) (G2 and H2) were detected in C7-PBS-injected mice (G1 and G2) and C7-TDP-43 PFFs-injected mice (H1 and H2). I. The CMCT was increased in C7-TDP-43 PFFs-injected mice compared to C7-PBS-injected mice at different time points post injection. J. The amplitude of cMEP was decreased in C7-TDP-43 PFFs-injected Atg5 $5^{+/}$mice compared to MI-PBS-injected Atg5 $5^{+/}$mice at different time points post injection. K-L. At the end-stage of MI-TDP-43 PFFs-injected Atg $5^{+/}$mice, H\&E staining of right biceps (K) showed muscle atrophy, round muscle fibers, and muscle fibers with central nuclei, while the left biceps (L) showed normal skeletal muscle morphology. Scale bar $=25 \mu \mathrm{m}$.

\section{M1- and C7-TDP-43 PFFs-injected Atg5+/- mice exhibit motor impairments}

We further investigated whether M1- and C7-TDP-43 PFFs-injected Atg5 $5^{+/}$mice exhibit ALS-like symptoms. First, the lifespan of TDP-43 PFFs-injected Atg5 $5^{+/-}$mice was significantly shorter compared with PBS-injected controls, and the Kaplan-Meier curve showed overall survival in Figure 7A. To assess the motor function of TDP-43 PFFs-injected Atg5 $5^{+/}$mice, we performed a series of behavioral tests. At approximately 8 weeks post-injection, C7-TDP-43 PFFs-injected Atg5+/- mice gradually lost weight (Figure 7B) and performed poorly on the rotarod test (Figure $7 \mathrm{C}$ ) and hanging wire test (Figure 7D), revealing a significant deficit in muscle strength, motor coordination, and balance. In addition, C7-TDP-43 PFFs-injected Atg5 ${ }^{+/}$mice showed significantly shorter stride length and wider base width compared to PBS-injected controls in the footprint test (Figure 6E-J). As for M1-TDP-43 PFFs-injected Atg5+- mice, they began to lose weight at around 10 weeks post-injection (Figure 7B), and they also showed deficient motor performance in the rotarod test and hanging wire test compared to age-matched PBS-injected Atg $5^{+/}$-mice (Figure $7 \mathrm{C}$ and 7D). However, the severity of motor disability was much higher in C7-TDP-43 PFFs-injected Atg5+\%-mice compared to age-matched C7-TDP-43 PFFs-injected Atg5 ${ }^{+/-}$mice (Figure 7C and 7D). Moreover, in the footprint test, M1-TDP-43 PFFs-injected Atg5+/- mice also showed significantly shorter stride length and wider base width similar to those found in C7-TDP-43 PFFs-injected Atg $5^{+/}$mice (Figure 7E-J). In conclusion, these changes suggest that the M1- and C7-TDP-43 PFFs-injected Atg5 $5^{+/-}$mice exhibited ALS-like symptoms, including short survival, weight loss, abnormal gait, and motor dysfunction.

\section{Discussion}

To investigate whether inoculation of TDP-43 PFFs can transmit pathological TDP-43 along corticospinal tract axons in vivo, we injected TDP-43 PFFs into the cortex or spinal cord anterior horn of Atg $5^{+/}$mice. The results show that unilateral injection of TDP-43 PFFs into the fifth layer of the left M1 or the left anterior horn of C7 of Atg5 $5^{+/-}$mice could induce the formation and spreading of pathological TDP-43 along the corticospinal tract. Moreover, we also found the presence of TDP-43 pathology in our animal models at CA1 region of hippocampus and hypoglossal nucleus in M1-TDP-43 PFFs-injected Atg5 $5^{+/}$mice at $6 \mathrm{mpi}$ and in C7-TDP-43 PFFs-injected Atg $5^{+/}$mice at $5 \mathrm{mpi}$. This supports the hypothesis that TDP-43 pathology could spread via axonal pathways [10]. Correspondingly, the regions that TDP-43 pathology spread to are also affected in ALS patients. 

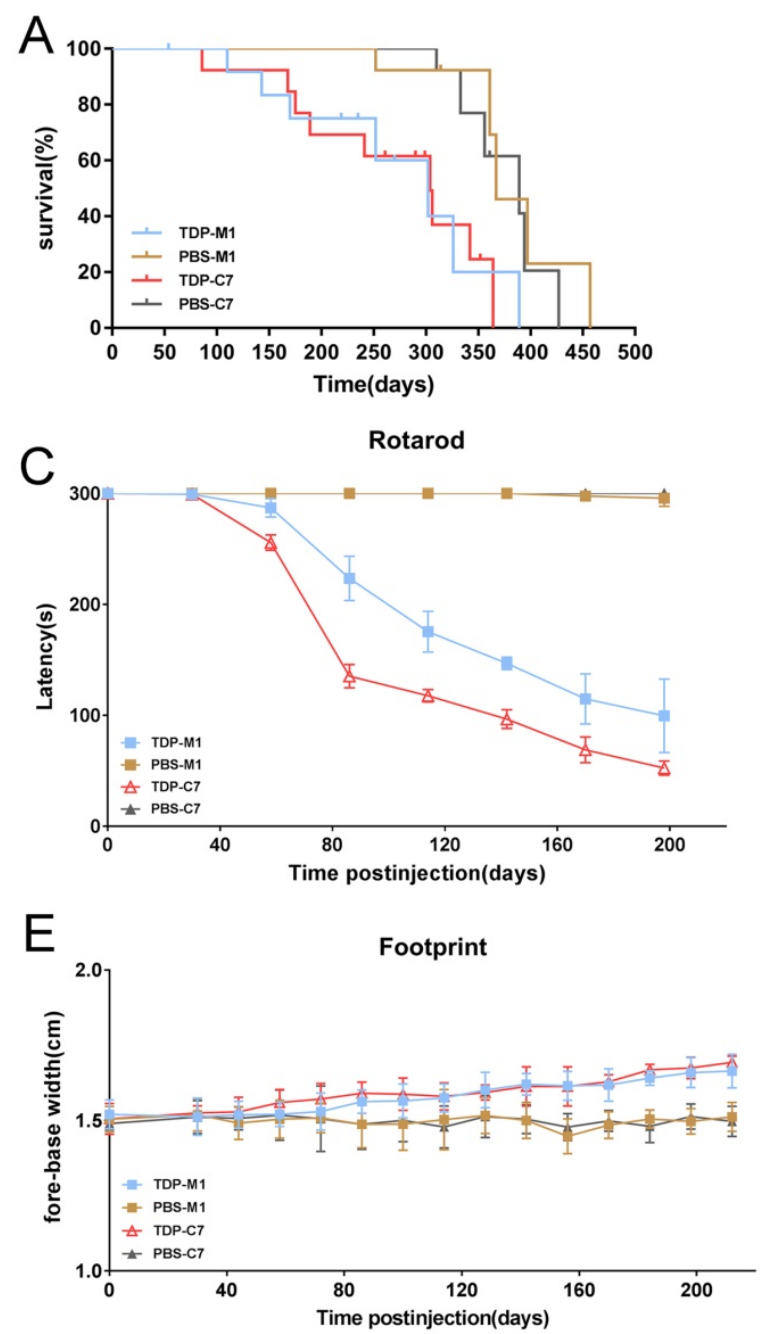

G
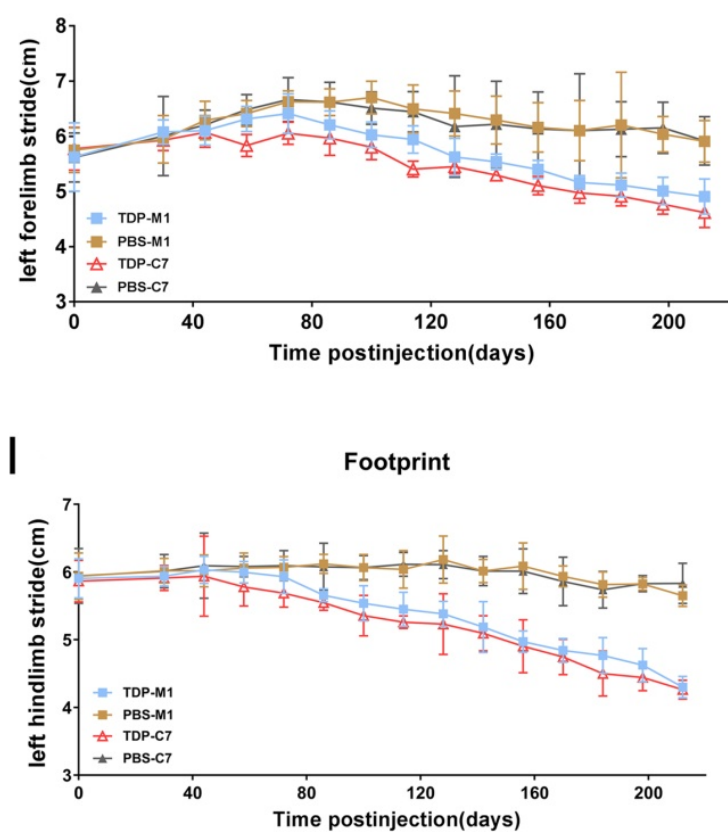
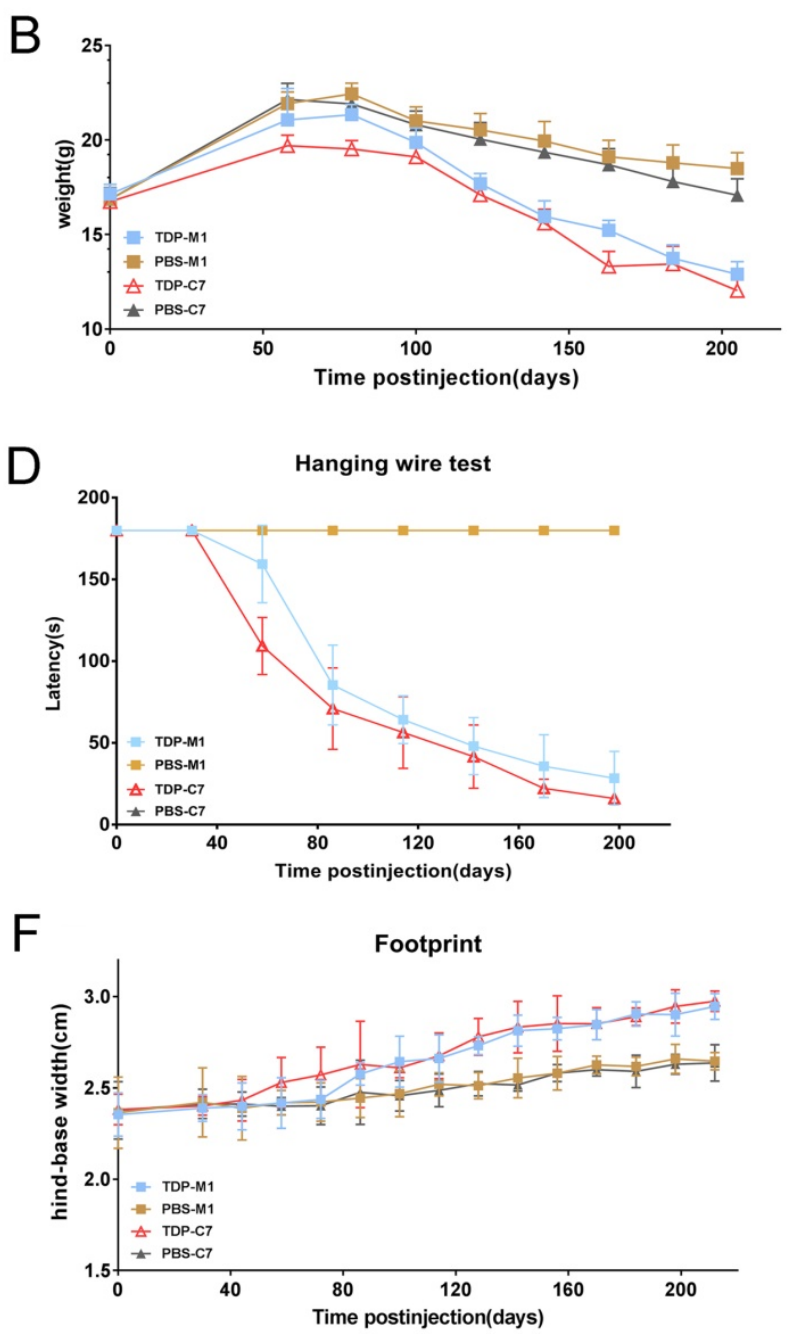

$\mathrm{H}$

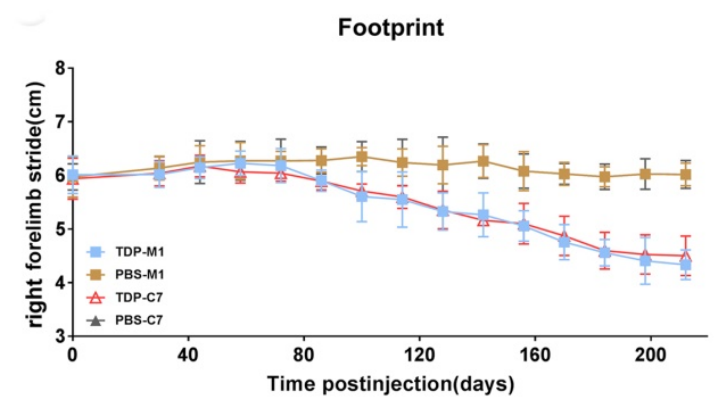

J

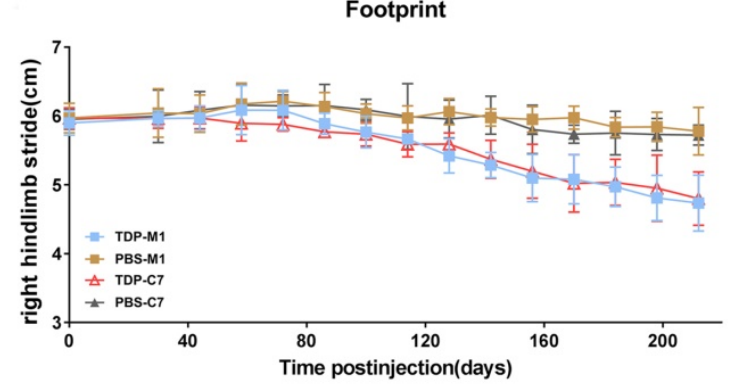

Figure 7. MI- and C7-TDP-43 PFFs-injected Atg5+/- mice developed motor impairments. A. Kaplan-Meier survival probability curve showed that the life span of TDP-43 PFFs-injected Atg5 $5^{+/-}$mice was shorter than PBS-injected Atg5 $5^{+/}$mice. B. M1- and C7-TDP-43 PFFs-injected Atg5 $5^{+/-}$mice developed meaningful weight decline at 8 weeks post injection 
compared to M1- and C7-PBS-injected mice, $p<0.001$. C. Rotarod test analysis of mice at various time post injection revealed that C7-TDP-43 PFFs-injected Atg5+/- mice had significant differences in rotarod latencies at 8 weeks post injection, MI-TDP-43 PFFs-injected Atg $5+/$ mice at 10 weeks compared to age-matched PBS-injected controls, $p<0.001$. D. Hanging wire test of mice at various times post injection revealed that C7-TDP-43 PFFs-injected Atg5 $5^{+/}$mice had significant differences in hanging wire latencies at 8 weeks post injection, MI-TDP-43 PFFs-injected Atg $5^{+/}$- mice at 10 weeks compared to age-matched PBS-injected controls, $p<0.001$. Longer latency indicates greater strength. E-J. The footprint test was performed on Atg $5^{+/-}$ mice every two weeks from 30 days to 200 days. C7-TDP-43 PFFs-injected mice showed shorter stride length (E-H) and wider base width (I and J) compared to C7-PBS-injected controls at 8 weeks post injection, $p<0.001$. MI-TDP-43 PFFs-injected Atg5 $5^{+/-}$mice performed meaningful changes at 10 weeks compared to age-matched PBS-injected controls. A two-way ANOVA followed by Bonferroni adjustment was used for statistical analysis. Data represent means \pm SD of four independent trials $(n=15$ mice/group).

It mimics the TDP-43 pathology in patients with ALS. These data indicate that TDP-43 seeding in vivo recapitulates ALS-like pathology. Furthermore, M1-TDP-43 PFFs-injected Atg5 ${ }^{+/-}$mice showed abnormal spontaneous activity in both sides of biceps brachialis, T10 paraspinals, tibialis anterior, and gastrocnemius muscles at around $5 \mathrm{mpi}$, and C7-TDP-43 PFFs-injected Atg5 ${ }^{+/-}$mice showed prolonged CMCT and decreased cMEP amplitude at around $2 \mathrm{mpi}$, which recapitulated the electrophysiologic changes found in ALS patients. On the other hand, these results also reflect the anterograde and retrograde spreading of pathological TDP-43.

It is known that autophagy is a vital degradation mechanism of dysfunctional organelles and large protein aggregates [21-24]. Autophagy also proved to have neuroprotective effects on the nervous system as a potent inhibitor of neurodegeneration, and impaired autophagy has been evidenced in various neurodegenerative diseases including ALS [25, 26]. Studies have shown that the preformed human TDP-43 aggregates were degraded via autophagy when transfected into murine NSC34 and N2a cells [27]. Also, autophagy inhibition promotes the accumulation of aggregated endogenous TDP-43 and $25-\mathrm{kDa}$ CTFs $[28,29]$. In addition, autophagy plays a critical part in TDP-43 aggregates clearance under physiological conditions [30]. Under pathological conditions, in vitro evidence has suggested that autophagy induction improves TDP-43 metabolism and clearance and prevents death in cultured neurons and astrocytes expressing mutant TDP-43 [31]. Numerous studies of TDP-43 proteinopathy animal models further support the neuronal protective effects of autophagy induction. In FTLD-U mouse and Drosophila models for TDP-43 proteinopathy, evidence shows that inducing autophagy effectively diminishes TDP-43 aggregation and finally rescues behavior deficits [32, 33]. Taken together, these studies demonstrate the relevance between autophagy and TDP-43 clearance. In our present study, we demonstrated the autophagy deficiency in M1, hippocampus, medulla oblongata, and cervical enlargement of Atg5 $5^{+/}$mice, and pTDP-43 levels were significantly increased in M1-TDP-43 PFFs-injected Atg $5^{+/-}$mice compared with age-matched PBS-injected Atg5 $5^{+/-}$mice. It indicated that autophagy deficiency may promotes the formation and spreading of pathological TDP-43 in vivo. Furthermore, our results showed that TDP-43 PFFs-injected Atg5 $5^{+/}$mice exhibits ALS-like neuropathology and motor phenotypes.

As we all know, SOD1 G93A transgenic mouse is a commonly used animal model for ALS. Previous studies have found increased autophagy in SOD1 G93A mice [34, 35]. It would be very helpful to observe the pathological and physiological changes in SOD1 G93A transgenic mice post injection of TDP-43 PFFs. We will shift our research focus from Atg5+/mice to SOD1 G93A transgenic mice in further study, thus making our results more convincing.

In conclusion, we provided the evidence that TDP-43 PFFs could induce the transmission of pathological TDP-43 along corticospinal tract axons in both an anterograde and retrograde manner in vivo. These data suggest that promoting autophagy and inhibiting cell-to-cell transmission of pathological TDP-43 may have therapeutic potential in the treatment of ALS.

\section{Abbreviations}

ALS: amyotrophic lateral sclerosis; CMCT: central motor conduction time; cMEP: cortical MEP; C7: the seventh cervical spinal cord segment; EMG: electromyography; FTLD: frontotemporal lobar degeneration; H\&E: Hematoxylin and eosin; LMN: lower motor neuron; MEPs: motor evoked potentials; mpi: months post-injection; MUAPs: motor unit action potentials; M1: primary motor cortex; PFFs: preformed fibrils; pTDP-43: phosphorylated TDP-43; pTDP-43-ir: pTDP-43-immunoreactive; sMEP: spinal MEP; TDP-43: TAR DNA-binding protein-43; UMN: upper motor neuron.

\section{Acknowledgments}

This work was supported by grants from the National Natural Science Foundation of China (No. $81671267,81471307)$. We thank all our collaborators at the First Affiliated Hospital of Zhengzhou University and Affiliated People's Hospital of Zhengzhou University for their assistance with technical support of EMGs and MEPs. We would like to thank Euni Wu for language editing of the manuscript.

\section{Author Contributions}

Design and concept: Xuejing Wang, Xuebing Ding, and Erxi Wu. 
Data acquisition and analysis: Rui Zhang, Yongkang Chen, Xinxin Wang, Haiyan Tian, Han Liu, Zhi Xiang, Dan Qi, Jason H. Huang, Erxi Wu, Xuebing Ding, and Xuejing Wang.

Manuscript drafting: Rui Zhang, Yongkang Chen, Xuebing Ding, and Xuejing Wang.

Manuscript revision: all.

\section{Competing Interests}

The authors have declared that no competing interest exists.

\section{References}

1. Li W, Liu Z, Sun W, et al. Mutation analysis of GLT8D1 and ARPP21 genes in amyotrophic lateral sclerosis patients from mainland China. Neurobiology of Aging. 2020; 85: 156.e151-156.e154.

2. Jiao B, Sun $Q$, Yuan $Z$, et al. Rare TBK1 variants in patients with frontotemporal dementia and amyotrophic lateral sclerosis in a Chinese cohort. Transl Neurodegener. 2018; 7: 31.

3. Hou L, Jiao B, Xiao T, et al. Screening of SOD1, FUS and TARDBP genes in patients with amyotrophic lateral sclerosis in central-southern China. Sci Rep. 2016; 6: 32478.

4. Yuan Z, Jiao B, Hou L, et al. Mutation analysis of the TIA1 gene in Chinese patients with amyotrophic lateral sclerosis and frontotemporal dementia. Neurobiol Aging. 2018; 64: 160 e169-160 e112.

5. Kwong LK, Neumann M, Sampathu DM, et al. TDP-43 proteinopathy: the neuropathology underlying major forms of sporadic and familial frontotemporal lobar degeneration and motor neuron disease. Acta Neuropathol. 2007; 114: 63-70.

6. Neumann M, Sampathu DM, Kwong LK, et al. Ubiquitinated TDP-43 in frontotemporal lobar degeneration and amyotrophic lateral sclerosis. Science. 2006; 314: 130-133.

7. Neumann M, Kwong LK, Lee EB, et al. Phosphorylation of S409/410 of TDP-43 is a consistent feature in all sporadic and familial forms of TDP-43 proteinopathies. Acta Neuropathol. 2009; 117: 137-149.

8. Burk K, Pasterkamp RJ. Disrupted neuronal trafficking in amyotrophic lateral sclerosis. Acta Neuropathol. 2019; 137: 859-877.

9. Brettschneider J, Arai K, Del TrediciK, et al. TDP-43 pathology and neuronal loss in amyotrophic lateral sclerosis spinal cord. Acta Neuropathol. 2014; 128 : 423-437.

10. Brettschneider J, Del TrediciK, Toledo JB, et al. Stages of pTDP-43 pathology in amyotrophic lateral sclerosis. Ann Neurol. 2013; 74: 20-38.

11. Johnson BS, Snead D, Lee JJ, et al. TDP-43 is intrinsically aggregation-prone, and amyotrophic lateral sclerosis-linked mutations accelerate aggregation and increase toxicity. J Biol Chem. 2009; 284: 20329-20339.

12. Furukawa $Y$, Kaneko $K$, Watanabe S, et al. A seeding reaction recapitulates intracellular formation of Sarkosyl-insoluble transactivation response element (TAR) DNA-binding protein-43 inclusions. J Biol Chem. 2011; 286: 18664-18672.

13. Nonaka T, Masuda-Suzukake $\mathrm{M}$, Arai $\mathrm{T}$, et al Prion-like properties of pathological TDP-43 aggregates from diseased brains. Cell Rep. 2013; 4: 124-134

14. Feiler MS, Strobel B, Freischmidt A, et al. TDP-43 is intercellularly transmitted across axon terminals. J Cell Biol. 2015; 211: 897-911.

15. Smethurst $P$, Newcombe J, Troakes $C$, et al. In vitro prion-like behaviour of TDP-43 in ALS. Neurobiol Dis. 2016; 96: 236-247.

16. Ishii $\mathrm{T}$, Kawakami E, Endo $\mathrm{K}$, et al. Formation and spreading of TDP-43 aggregates in cultured neuronal and glial cells demonstrated by time-lapse imaging. PLoS One. 2017; 12: e0179375.

17. Svahn AJ, Don EK, Badrock AP, et al. Nucleo-cytoplasmic transport of TDP-43 studied in real time: impaired microglia function leads to axonal spreading of TDP-43 in degenerating motor neurons. Acta Neuropathol. 2018; 136: 445-459.

18. Porta $\mathrm{S}, \mathrm{Xu} \mathrm{Y}$, Restrepo $\mathrm{CR}$, et al. Patient-derived frontotemporal lobar degeneration brain extracts induce formation and spreading of TDP-43 pathology in vivo. Nat Commun. 2018; 9: 4220.

19. Wils H, Kleinberger G, Janssens J, et al. TDP-43 transgenic mice develop spastic paralysis and neuronal inclusions characteristic of ALS and frontotemporal lobar degeneration. Proc Natl Acad Sci U S A. 2010; 107: 3858-3863.

20. Cardiff RD, Miller $\mathrm{CH}$, Munn RJ. Manual hematoxylin and eosin staining of mouse tissue sections. Cold Spring Harb Protoc. 2014; 2014: 655-658.

21. Rubinsztein DC. The roles of intracellular protein-degradation pathways in neurodegeneration. Nature. 2006; 443: 780-786.

22. Nikoletopoulou V, Tavernarakis N. Regulation and Roles of Autophagy at Synapses. Trends Cell Biol. 2018; 28: 646-661.

23. Mizushima N. A brief history of autophagy from cell biology to physiology and disease. Nat Cell Biol. 2018; 20: 521-527.
24. Wang $\mathrm{R}$, Tan $\mathrm{J}$, Chen $\mathrm{T}$, et al. ATP13A2 facilitates HDAC6 recruitment to lysosome to promote autophagosome-lysosome fusion. J Cell Biol. 2019; 218: 267-284.

25. Madeo F, Eisenberg T, Kroemer G. Autophagy for the avoidance of neurodegeneration. Genes Dev. 2009; 23: 2253-2259.

26. Ramesh N, Pandey UB. Autophagy Dysregulation in ALS: When Protein Aggregates Get Out of Hand. Front Mol Neurosci. 2017; 10: 263.

27. Cascella R, Fani G, Capitini C, et al. Quantitative assessment of the degradation of aggregated TDP-43 mediated by the ubiquitin proteasome system and macroautophagy. FASEB J. 2017; 31: 5609-5624.

28. Filimonenko M, Stuffers S, Raiborg C, et al. Functional multivesicular bodies are required for autophagic clearance of protein aggregates associated with neurodegenerative disease. J Cell Biol. 2007; 179: 485-500.

29. Wang X, Fan H, Ying Z, et al. Degradation of TDP-43 and its pathogenic form by autophagy and the ubiquitin-proteasome system. Neurosci Lett. 2010; 469: 112-116.

30. Scotter EL, Vance C, Nishimura AL, et al. Differential roles of the ubiquitin proteasome system and autophagy in the clearance of soluble and aggregated TDP-43 species. J Cell Sci. 2014; 127: 1263-1278.

31. Barmada SJ, Serio A, Arjun A, et al. Autophagy induction enhances TDP43 turnover and survival in neuronal ALS models. Nat Chem Biol. 2014; 10: 677-685.

32. Cheng CW, Lin MJ, Shen CK. Rapamycin alleviates pathogenesis of a new Drosophila model of ALS-TDP. J Neurogenet. 2015; 29: 59-68.

33. Wang IF, Tsai KJ, Shen CK. Autophagy activation ameliorates neuronal pathogenesis of FTLD-U mice: a new light for treatment of TARDBP/TDP-43 proteinopathies. Autophagy. 2013; 9: 239-240.

34. Morimoto N, Nagai M, Ohta $\mathrm{Y}$, et al. Increased autophagy in transgenic mice with a G93A mutant SOD1 gene. Brain Research. 2007; 1167: 112-117.

35. Li L, Zhang $X$, Le W. Altered macroautophagy in the spinal cord of SOD1 mutant mice. Autophagy. 2008; 4: 290-293. 